\title{
A pedigree-based prediction model identifies carriers of deleterious de novo mutations in families with Li-Fraumeni syndrome
}

\author{
Fan Gao, ${ }^{1,2,11}$ Xuedong Pan, ${ }^{1,3,11}$ Elissa B. Dodd-Eaton, ${ }^{1}$ Carlos Vera Recio, ${ }^{1}$ \\ Matthew D. Montierth, ${ }^{1,4}$ Jasmina Bojadzieva, ${ }^{5}$ Phuong L. Mai, ${ }^{6,12}$ Kristin Zelley, ${ }^{7}$ \\ Valen E. Johnson, ${ }^{3}$ Danielle Braun, ${ }^{8,9}$ Kim E. Nichols, ${ }^{7,13}$ Judy E. Garber, ${ }^{10}$ \\ Sharon A. Savage, ${ }^{6}$ Louise C. Strong, ${ }^{5}$ and Wenyi Wang ${ }^{1}$
}

${ }^{1}$ Department of Bioinformatics and Computational Biology, The University of Texas MD Anderson Cancer Center, Houston, Texas 77030, USA; ${ }^{2}$ Department of Statistics, Rice University, Houston, Texas 77005, USA; ${ }^{3}$ Department of Statistics, Texas A\&M University, College Station, Texas 77843, USA; ${ }^{4}$ Quantitative and Computational Biosciences, Baylor College of Medicine, Houston, Texas 77030, USA; ${ }^{5}$ Department of Genetics, The University of Texas MD Anderson Cancer Center, Houston, Texas 77030, USA; ${ }^{6}$ Clinical Genetics Branch, Division of Cancer Epidemiology and Genetics, National Cancer Institute, Bethesda, Maryland 20892, USA; ${ }^{7}$ Division of Oncology, Children's Hospital of Philadelphia, Philadelphia, Pennsylvania 19104, USA; ${ }^{8}$ Department of Biostatistics, Harvard T.H. Chan School of Public Health, Boston, Massachusetts 02115, USA; ${ }^{9}$ Department of Data Sciences, DanaFarber Cancer Institute, Boston, Massachusetts 02215, USA; ${ }^{10}$ Dana-Farber Cancer Institute and Harvard Medical School, Boston, Massachusetts 02215, USA

\begin{abstract}
De novo mutations (DNMs) are increasingly recognized as rare disease causal factors. Identifying DNM carriers will allow researchers to study the likely distinct molecular mechanisms of DNMs. We developed Famdenovo to predict DNM status (DNM or familial mutation [FM]) of deleterious autosomal dominant germline mutations for any syndrome. We introduce Famdenovo.TP53 for Li-Fraumeni syndrome (LFS) and analyze 324 LFS family pedigrees from four US cohorts: a validation set of 186 pedigrees and a discovery set of 138 pedigrees. The concordance index for Famdenovo.TP53 prediction was 0.95 (95\% Cl: $[0.92,0.98])$. Forty individuals $(95 \% \mathrm{Cl}:[30,50])$ were predicted as DNM carriers, increasing the total number from 42 to 82. We compared clinical and biological features of FM versus DNM carriers: (1) cancer and mutation spectra along with parental ages were similarly distributed; (2) ascertainment criteria like early-onset breast cancer (age 20-35 yr) provides a condition for an unbiased estimate of the DNM rate: 48\% (23 DNMs vs. 25 FMs); and (3) hotspot mutation R248W was not observed in DNMs, although it was as prevalent as hotspot mutation R248Q in FMs. Furthermore, we introduce Famdenovo.BRCA for hereditary breast and ovarian cancer syndrome and apply it to a small set of family data from the Cancer Genetics Network. In summary, we introduce a novel statistical approach to systematically evaluate deleterious DNMs in inherited cancer syndromes. Our approach may serve as a foundation for future studies evaluating how new deleterious mutations can be established in the germline, such as those in TP53.
\end{abstract}

[Supplemental material is available for this article.]

De novo mutations (DNMs) are defined as germline variants (mutations) occurring in the carrier but not in either of the carrier's parents (Rahbari et al. 2016). In humans, dozens of DNMs (Nachman and Crowell 2000; Kondrashov 2003; Conrad et al. 2006; Lynch 2010; Kong et al. 2012; Lipson et al. 2015) occur throughout the genome of each newborn. DNMs are considered a major cause for the occurrence of rare, early-onset reproductively lethal diseases (Acuna-Hidalgo et al. 2016). Initial analyses have shown that the rate of germline DNMs increases with paternal

\footnotetext{
${ }^{11}$ These authors contributed equally to this work.

Present addresses: ${ }^{12}$ Magee-Womens Hospital of the University of Pittsburgh Medical Center, Pittsburgh, PA 15213, USA; ${ }^{13}$ Division of Cancer Predisposition, St. Jude Children's Research Hospital, Memphis, TN 38105, USA

Corresponding author: wwang7@mdanderson.org

Article published online before print. Article, supplemental material, and publication date are at http://www.genome.org/cgi/doi/10.1101/gr.249599.119. Freely available online through the Genome Research Open Access option.
}

(Kong et al. 2012) and maternal age (Wong et al. 2016), plus, DNMs are correlated to a number of factors (Battle and Montgomery 2014), including the time of replication (Francioli et al. 2015), the rate of recombination (Francioli et al. 2015), GC content (Michaelson et al. 2012), and DNA hypersensitivity (Michaelson et al. 2012). DNM carriers and their associated factors have always been a focus of genetics research because of their crucial role in the evolution of species (Goldmann et al. 2019).

Not only are DNMs the raw materials of evolution, but they may also shape and determine disease susceptibility (de Ligt et al. 2012; De Rubeis et al. 2014; Iossifov et al. 2014; Robinson et al. 2014; Krumm et al. 2015). DNMs have been recognized as causal factors for rare diseases, such as retinoblastoma and neurofibromatosis, where the de novo rates are as high as 90\% (Dryja

(c) 2020 Gao et al. This article, published in Genome Research, is available under a Creative Commons License (Attribution-NonCommercial 4.0 International), as described at http://creativecommons.org/licenses/by-nc/4.0/. 
et al. 1997) and 50\% (Evans et al. 2010; Jett and Friedman 2010), respectively. Li-Fraumeni syndrome (LFS) is an autosomal-dominant cancer predisposition syndrome that commonly manifests as soft tissue and bone sarcomas, breast cancer, brain tumors, leukemia, and adrenal cortical carcinomas (Nichols et al. 2001; Olivier et al. 2003) and is associated with germline mutations in the TP53 tumor suppressor gene (Malkin et al. 1990; Correa 2016), of which 7\%-20\% are de novo (Gonzalez et al. 2009). Clinically, it is of interest to identify de novo carriers of a TP53 germline mutation, who may otherwise go unnoticed until they or their offspring develop multiple cancers, to provide them with information on current standard LFS screening and surveillance protocols. Understanding the cause of de novo mutations in cancer genes like TP53 will help develop strategies for early identification, which will have a novel and significant impact on the field of human genetics.

Predicting a DNM in a gene is not trivial when the parental mutation statuses are unknown. Incomplete family history may skew observational criteria and the prediction may suffer from low sensitivity and specificity. Mendelian risk prediction models (Chen et al. 2004) have been used successfully for accurate prediction of deleterious mutation status in familial breast and ovarian (Euhus et al. 2002), bowel (Chen et al. 2006b), and pancreatic (Wang et al. 2007) cancers, melanoma (Wang et al. 2010), and most recently in LFS (Peng et al. 2017; Renaux-Petel et al. 2018). We developed a novel statistical approach, Famdenovo, to predict a mutation carrier's DNM status, i.e., whether the carrier's deleterious mutation was due to familial inheritance or alternatively arose from a new mutation in that individual. Built on the principles of Mendelian models, Famdenovo predicts a new biological outcome, DNM status. For each particular set of gene(s) of interest, three sets of input parameters need to be estimated from a population study in order to build Famdenovo: the penetrance, the allele frequency, and the de novo mutation rate (percent of de novo mutations among all germline mutations) of the deleterious alleles.

DNMs are rare and therefore require a large amount of high-quality data to be studied thoroughly. We introduce Famdenovo.TP53, which was modeled using input parameters estimated from published studies and validated on a large cohort consisting of 324 LFS family cohorts that were collected from four major cancer centers in the United States, with TP53 genetic testing results available in at least one trio per family in 186 families. The computer-based and data-driven identification of deleterious DNM carriers in TP53, who are otherwise hidden in a wide population, enabled a new perspective of DNMs that is geneand disease-centric. In addition, we introduce Famdenovo.BRCA for hereditary breast and ovarian cancer syndrome (HBOC) and apply it to a small set of family data from the Cancer Genetics Network (CGN).

\section{Results}

\section{Famdenovo for predicting the deleterious DNM status among} mutation carriers

Famdenovo is based on widely used Mendelian models (Parmigiani et al. 1998; Chen et al. 2004, 2006b; Wang et al. $2007,2010)$ and calculates the probability that a deleterious germline mutation is de novo. The de novo probability of interest can be written as the $\operatorname{Pr}$ (father is a noncarrier, mother is a noncarrier | child is a mutation carrier, family history). We calculate this probability as a function, $\mathrm{g}(c, f, m)$, of pedigree-based carrier probabili- ties for the child $(c)$, father $(f)$, and mother $(m)$, respectively. We derived the $\mathrm{g}()$ function using the Bayes' rule (as detailed in the Methods section) and obtained input values using Mendelian models as the corresponding marginal probabilities for one individual's $c$, $f$, and $m$, respectively. Each of the marginal probabilities is calculated using the Elston-Stewart algorithm (Fernando et al. 1993). Famdenovo then applies the Bayes' rule to estimate de novo probabilities in deleterious mutations. Famdenovo can be adapted for specific disease-gene associations by setting its input parameters accordingly: the penetrance of the diseasegene, the allele frequency of the gene(s), and the de novo mutation rate. We have incorporated well-validated parameter estimates in Famdenovo.TP53 for LFS-TP53 (Peng et al. 2017) and Famdenovo.BRCA for HBOC-BRCA1/2 (Chen et al. 2006a; Parmigiani et al. 2007).

Figure 1 depicts three scenarios of a hypothetical pedigree resulting in different DNM probabilities in TP53 given by Famdenovo.TP53. Figure 1A shows a pedigree with four cancer cases: two soft tissue sarcomas (STS) at 25 and $27 \mathrm{yr}$ old, one breast cancer at $40 \mathrm{yr}$ old, and another STS at $20 \mathrm{yr}$ old. The arrow points to the counselee who is a TP53 mutation carrier, and the probability of the mutation being de novo for the counselee is 0.0033 . It is likely that this is a familial mutation inherited from the counselee's mother. Figure 1B shows a similar pedigree with three cancer patients, with one less affected relative in the maternal branch of the counselee compared with Figure 1A, and with an older age of diagnosis for the counselee's mother, which increases the predicted probability of the mutation being de novo. The de novo probability given by Famdenovo.TP53 is 0.47 . Figure 1C shows a similar pedigree with only two cancer cases in the counselee and counselee's offspring, compared with Figure 1B. Because there are no longer any cancer patients in the older generations and the counselee's siblings are all healthy, it is most likely that this mutation is de novo. Famdenovo.TP53 predicted this mutation to be a DNM, based on the 0.89 probability of the mutation being de novo.

\section{Validation of Famdenovo.TP53 in individuals with known DNM status in TP53}

We evaluated the performance of Famdenovo.TP53 based on germline TP53 tested individuals with known DNM status from the four cohorts. Among the 324 LFS family pedigrees we collected, 186 families had a known DNM status and 138 had an unknown DNM status (Fig. 2; Supplemental Table S1). Table 1 provides a summary of the demographics for all four study cohorts. The largest set of DNM carriers was contributed by the MD Anderson (MDA) cohort. In total, there were 42 known DNM carriers and 144 families with known FM carriers.

We used observed:estimated ratios (OEs) and receiver operating characteristic (ROC) curves to evaluate the calibration and discrimination of our model. Figure 3 shows the ROC curves for the validation set from the four cohorts combined. The total sample size for the validation was 186 families; the corresponding area under the curve (AUC, or concordance index) was 0.95 (95\% CI: $[0.92,0.98])$, which demonstrated that Famdenovo.TP53 performed well in discriminating DNM carriers from FM carriers. The calibration was satisfactory, with some underprediction and an OE of 1.33 (95\% CI: [1.09, 1.63]).

We also compared our method with two simple criterionbased prediction models that do not require the collection of extended pedigree data (Table 2). Criterion 1 predicts a mutation to 


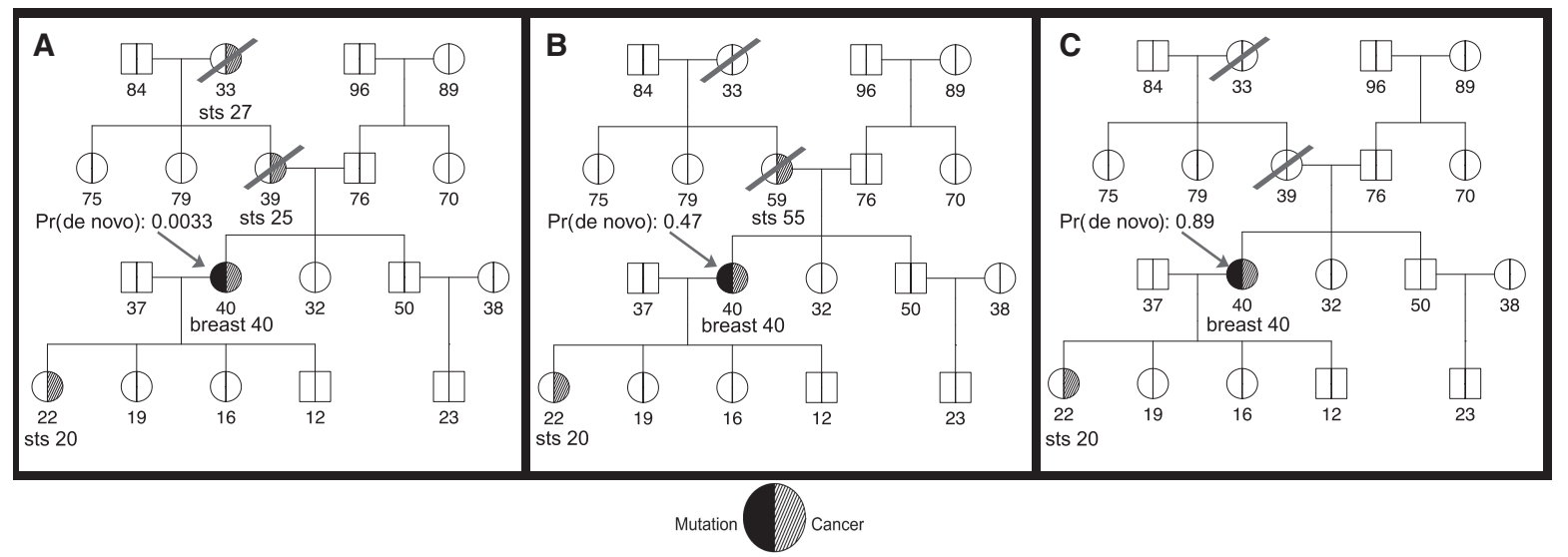

Figure 1. Illustration of clinical counseling using Famdenovo based on family history. The number below each individual is the age at last contact (healthy or affected with cancer). The arrow points to the counselee who is known to be a TP53 mutation carrier. The de novo probabilities shown are given by Famdenovo.TP53. (A) A pedigree with four cancer patients. (B) A pedigree with three cancer patients. (C) A pedigree with two cancer patients.

be de novo if the mutation carrier does not have a parent diagnosed with cancer before a cutoff age; e.g., 45 or 60 . Criterion 2 predicts a mutation to be de novo if, in addition to criterion 1 , neither of the grandparents was diagnosed with cancer before the cutoff age. Criterion 1 is more relaxed than criterion 2. Figure 3 shows that both criteria (over a range of cutoff ages) fall outside of the $95 \%$ confidence interval band of our method. Neither of the criteria is able to reach the full range of the ROC curve. Criterion 2 follows the same trend as criterion 1. Moreover, for each criterion, we calculated a partial AUC area with cutoff ages from 45 to 65 , which were then rescaled to the full range of 0 to 1 . The rescaled AUC for criterion 1, the better performing of the two criteria, is still much lower than Famdenovo.TP53, with a difference of 0.14 (95\% CI: $[0.054,0.20])$ (Table 2). Our results demonstrate that Famdenovo.TP53 outperforms criterionbased prediction models and that extended pedigrees with cancer diagnosis information are needed for the accurate identification of DNM carriers.

We evaluated the sensitivity and specificity of Famdenovo.TP53 under various prediction cutoff values using the validation set (Supplemental Table S2) in order to identify an optimal classification cutoff for this data set. If the predicted probability was equal to or above the prediction cutoff, the individual was classified as a DNM carrier. As the cutoff probability increases, the sensitivity decreases and specificity increases. We chose a cutoff of 0.2 , which provided a good tradeoff of a sensitivity of 0.76 and a specificity of 0.93 (Fig. 4A). Maintaining a high specificity is essential to allow for downstream clinical interpretation of de novo mutations. Using the observed deleterious DNMs in our TP53 clinical cohorts, we estimated that Famdenovo.TP53, at a cutoff $=0.2$, will achieve a positive predictive value (PPV) of 0.78 and a negative predictive value (NPV) of 0.92 . Without using pedigree information and model-based analysis like Famdenovo.TP53, criterion 1 will only achieve a PPV of 0.63 and an NPV of 0.92 (cutoff age $=65$, sensitivity $=0.8$, and specificity $=0.8$ ).

We also conducted a sensitivity analysis to evaluate how the pedigree size affects the validation results. The range of pedigree sizes for each of the four cohorts is listed in Table 1 (see also Supplemental Fig. S1). We divided the 186 families in the validation data set into two groups: large families and small families, using the median size of all families $(n=29)$ as the cutoff size. We obtained similar results under the two scenarios (small families: AUC 0.96, 95\% CI: [0.91, 0.99], large families: AUC 0.94, 95\% CI: $[0.89,0.98])$. These results support the robustness of our model by showing that Famdenovo is capable of accurately predicting de novo TP53 mutations in a range of family sizes.

We further conducted a sensitivity analysis on the two input parameters: the prior values for the deleterious allele frequency 
Table 1. Summary of demographic information for all the families with TP53 mutation carriers from the four cohorts

\begin{tabular}{|c|c|c|c|c|c|c|}
\hline \multirow[t]{2}{*}{ Cohort } & \multirow[b]{2}{*}{ No. of families } & \multirow[b]{2}{*}{140} & \multicolumn{2}{|c|}{ Individuals per family } & \multicolumn{2}{|c|}{ Age at first diagnosis } \\
\hline & & & Mean & 45 & Mean & 42.3 \\
\hline \multirow[t]{3}{*}{ MDA } & Max. family size & 151 & Standard deviation & 31 & Standard deviation & 22.2 \\
\hline & Min. family size & 4 & & & & \\
\hline & No. of families & 78 & Mean & 24 & Mean & 33.9 \\
\hline \multirow[t]{3}{*}{$\mathrm{NCl}$} & Max. family size & 130 & Standard deviation & 17 & Standard deviation & 22.1 \\
\hline & Min. family size & 3 & & & & \\
\hline & No. of families & 91 & Mean & 34 & Mean & 38.9 \\
\hline \multirow[t]{3}{*}{$\mathrm{DFCl}$} & Max. family size & 107 & Standard deviation & 25 & Standard deviation & 20.5 \\
\hline & Min. family size & 3 & & & & \\
\hline & No. of families & 15 & Mean & 26 & Mean & 43.4 \\
\hline \multirow[t]{2}{*}{$\mathrm{CHOP}$} & Max. family size & 45 & Standard deviation & 12 & Standard deviation & 25.2 \\
\hline & Min. family size & 13 & & & & \\
\hline
\end{tabular}

and the DNM rate. These values are expected to be dominated by family history data that provide strong information during the calculation of posterior probabilities. Tuning the two parameters barely influenced the AUC or OE (Supplemental Table S3).

\section{Discovery in individuals with unknown DNM status using Famdenovo}

We applied Famdenovo.TP53 to the 138 families with unknown DNM status in the discovery set from the same four cohorts. Figure 4B provides the probability distribution of predicted DNM carriers at a cutoff of 0.2 . In total, 40 (95\% CI: [30, 50]) out of the 138 families were predicted to be de novo. The number of predicted de novo families for MDA, NCI, DFCI, and CHOP were 23 out of 58 (95\% CI: [16, 30]), seven out of 12 (95\% CI: [4, 10]), seven out of 61 (95\% CI: [3, 12]), and three out of seven (95\% CI: [1, 5]), respectively. MDA and CHOP had higher percentages of DNMs, likely due to differences in ascertainment across cohorts. Sensitivity analyses using cutoffs from 0.05 to 0.3 gave consistent DNM results (Supplemental Table S4).

\section{Association of deleterious DNMs in TP53 with clinical outcomes}

Combining the validation and discovery sets resulted in a total of 82 DNMs from 82 families and 450 FMs from 242 families, which equipped our study with a sample size that was meaningful to evaluate associations between mutation status and a diverse range of individual outcomes: sex, cancer types, multiple primary cancers, age of cancer diagnosis, parental ages, and types of mutation.

Comparing DNM to FM carriers in 397 cancer patients (Supplemental Table S5), we observed more females than males (odds ratio $[\mathrm{OR}]=1.78,95 \% \mathrm{CI}$ : $[1.01,3.13]$ ). Considering the strong association between sex and breast cancer, we asked whether this significant finding comes from ascertainment bias. We performed another test within the probands; i.e., the index person that brings the family into the research cohort. This time, in contrast, we found no sex differences (34\% DNMs in female and $32 \%$ DNMs in male, OR $=1.08,95 \% \mathrm{CI}$ : $[0.58,2.03])$. All but two DNM carriers were probands. Therefore, we infer that the former significant finding was due to an imbalanced addition of FM carriers, who were relatives of the probands, to the female and male categories, i.e., a numerical artifact. In the following association analyses, we present comparisons within the probands only.

Among 233 probands with cancer information (Supplemental Table S6), we observed an OR=1.9 (95\% CI: $[1.09,3.33])$ for DNMs in breast cancer as compared to other cancer types. We fur- ther divided the probands into three age categories: [0, 20], [20, 35], and 35+, using age of last contact. We observed, within the [20, 35] age category, an $\mathrm{OR}=5.98$ (95\% CI: $[1.81,19.76])$ for DNMs in breast cancer as compared to other cancer types, whereas a similar test in the age $35+$ category was not statistically significant. This association is again due to ascertainment of DNMs through patients with early-onset breast cancer and not with other cancer types (see Supplemental Table S7 for commonly used ascertainment criteria; Li and Fraumeni 1969; Tinat et al. 2009). Within the [20, 35] age category and probands with breast cancer, where the ascertainment of each individual is consistent, we found 23 DNM carriers (29\% of all proband DNM carriers) and $25 \mathrm{FM}$ carriers (16\% of all proband $\mathrm{FM}$ carriers), resulting in a $48 \%(\mathrm{SD}=7.2 \%) \mathrm{DNM}$ rate. This high proportion of DNMs is also observed within the validation set only ( $n=11$ vs. 8 ) (Supplemental Table S6C). Sensitivity analyses using various thresholds of age of last contact showed consistent results (Supplemental Table S6D), supporting the robustness of this finding.

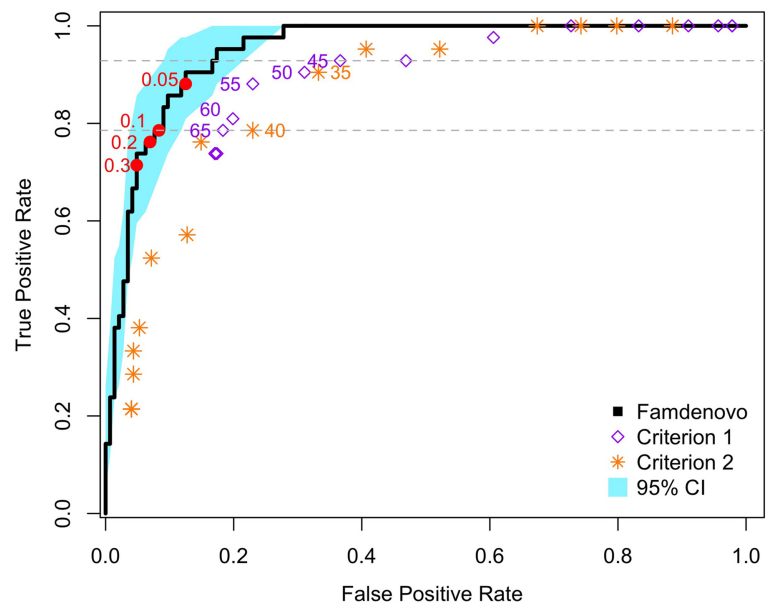

Figure 3. ROC curves for the validation of Famdenovo.TP53. The $x$-axis is the false positive rate (i.e., 1 - specificity), and the $y$-axis is the true positive rate (i.e., sensitivity). The black curve corresponds to the results of Famdenovo.TP53 on the 186 families in the validation set, and the blue shade shows the $95 \%$ confidence interval. The red circles represent the different de novo cutoff probabilities for the model. The purple diamonds correspond to the results of criterion 1 at a cutoff age (provided next to the diamonds). The orange stars represent the result of criterion 2 at a cutoff age (provided next to the stars). The gray dotted lines show the range of cutoff ages, 45-65, where a partial AUC is calculated for criterion 1. 
Table 2. Comparison of AUCs of Famdenovo.TP53 and various criterion-based prediction models, where $95 \% \mathrm{Cls}$ were obtained by bootstrapping

\begin{tabular}{lcc}
\hline AUC & Mean & $95 \% \mathrm{Cl}$ \\
\hline Famdenovo.TP53 & 0.95 & {$[0.92,0.98]$} \\
Criterion 1 & 0.82 & {$[0.78,0.87]$} \\
Criterion 2 & 0.80 & {$[0.77,0.84]$} \\
Famdenovo - criterion 1 & 0.14 & {$[0.054,0.20]$} \\
Famdenovo - criterion 2 & 0.15 & {$[0.087,0.20]$} \\
\hline
\end{tabular}

We evaluated the association between the DNM status and the risk of developing subsequent multiple primary cancers (MPC) within the probands. Supplemental Table S8A shows that more patients with DNMs presented multiple primary than single primary cancers $(\mathrm{OR}=1.77,95 \% \mathrm{CI}$ : [1.01, 3.11]). Similarly, we attribute such association to the ascertainment practice, i.e., ascertaining patients with a personal history of multiple primary cancers (at least one cancer is at an early onset) is part of the clinical criteria used for patient ascertainment in our study cohorts (Li and Fraumeni 1969; Tinat et al. 2009). In an attempt to adjust for ascertainment to make the DNM and FM carriers comparable, we looked within the $[20,35]$ age category at probands with MPC. We found 17 DNM carriers and $22 \mathrm{FM}$ carriers, resulting in $44 \%$ of DNMs within this combined cohort population ( $\mathrm{SD}=7.9 \%$ ) (Supplemental Table $\mathrm{S} 8 \mathrm{~B})$. This high proportion is also observed with the validation set ( $n=10$ vs. 8) (Supplemental Table S8C). This result is congruent with the previous observation of a high DNM rate in probands with early-onset breast cancer.

Figure 5A shows the distributions of cancer diagnosis age as stratified by cancer type and sex for the combined set. We observed DNMs in all of the eight LFS spectrum cancers: breast, brain, leukemia, choroid, adrenocortical carcinoma, osteosarcoma, soft tissue sarcoma, and lung, as well as other invasive cancers that are not within the LFS spectrum. Other than breast cancer, most cancer types have both female and male DNM carriers, with the exceptions of lung cancer and leukemia. For lung cancer, DNMs were only presented in females $(n=5$, mutation type: NC_000017.11:g.7675232G $>$ A [p.S127F], NC_000017.11:g.76751 57G >A [p.P152L], NC_000017.11: g.7674894_7674896delinsTGG [p.R213 W], NC_000017.11:g.7674230C > T [p.G 245S], NC_000017.11:g.7670699C > T [p.R337H]) (Supplemental Table S9) and not males. For leukemia, DNMs also were only presented in females $(n=2$, mutation type: NC_000017.11:g.76737 76G >A [p.R282W]) (Supplemental Table S9). We found no difference in ages of diagnosis between DNM and FM carriers across cancer types, including breast cancer (Fig. 5A).

We also investigated whether the parental ages (paternal or maternal) were different among TP53 DNM and FM carriers. Parental age refers to the age of the parent at the time of child birth. Literature on the origins of DNMs suggested their occurrence rate across the genome was correlated with paternal age (Kong et al. 2012). Little is known about occurrence of deleterious DNMs within a given gene with regard to parental ages. Here, parental age is obtained by calculating the difference between the birth year of the child and the parent. Figure 5, B and C, shows the distributions for the combined set. We observed that both paternal age $($ mean $=$ $29, \mathrm{SD}=5.8$ ) and maternal age (mean $=27, \mathrm{SD}=5.0$ ) were similarly distributed between DNMs and FM carriers. This is consistent with
A
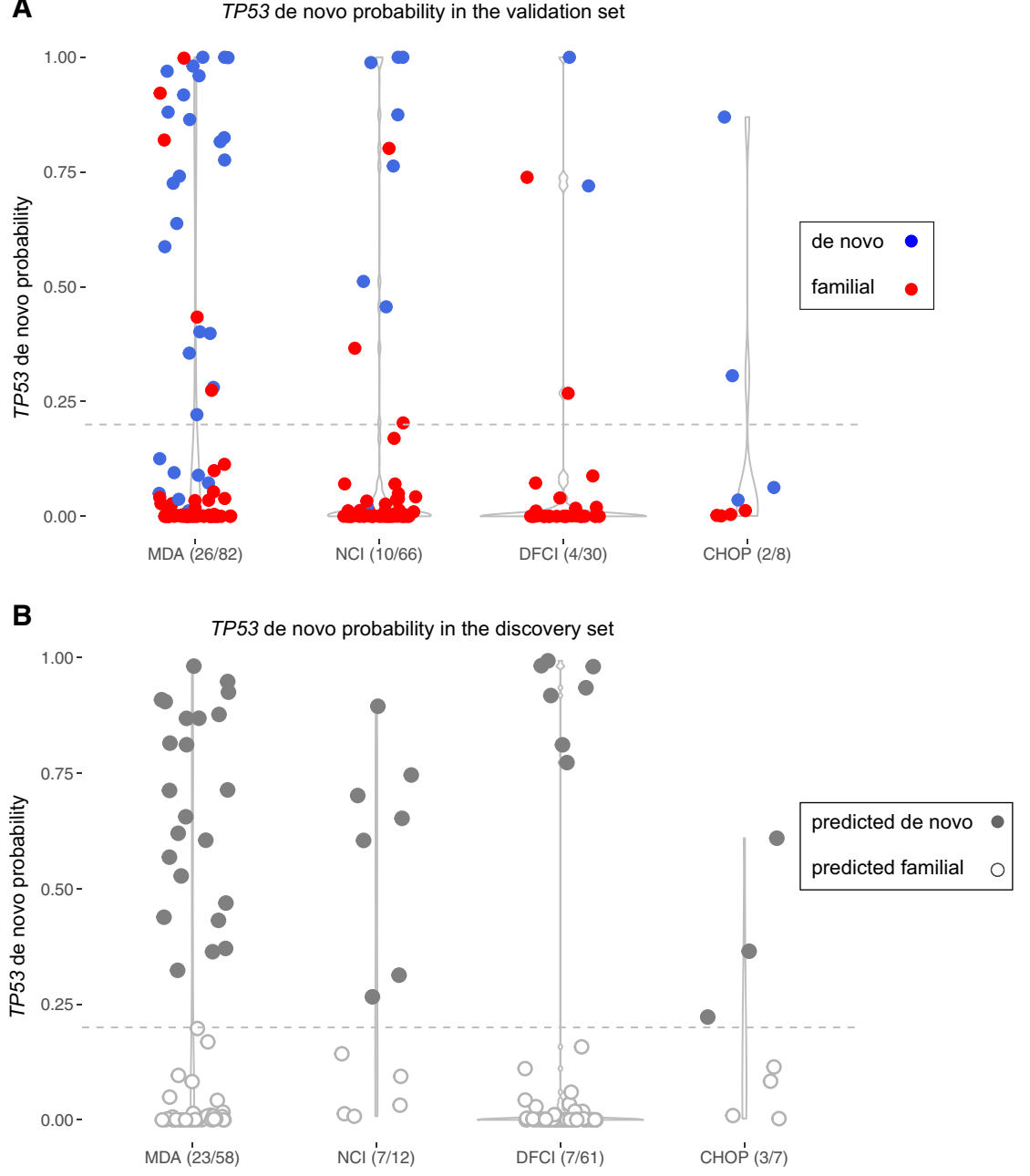

Figure 4. Distributions of the de novo probabilities in validation and discovery sets in the four cohorts. The $y$-axis is the predicted de novo probability by Famdenovo.TP53. The width of each column changes over the $y$-axis, representing the percent of individuals who have the corresponding range of probability values. The individuals with points above the gray line (cutoff $=0.2$ ) were predicted to be DNM carriers. The $x$-axis gives the names of the cohorts and the corresponding predicted numbers of DNM carriers out of the total number of individuals. $(A)$ In the validation set, the individuals with blue dots were true DNM carriers, and those with red dots were true FM carriers. $(B)$ In the discovery set, the individuals with solid gray dots were predicted to be DNM carriers, and those with empty dots were predicted to be FM carriers.

\section{Genome Research}

www.genome.org 


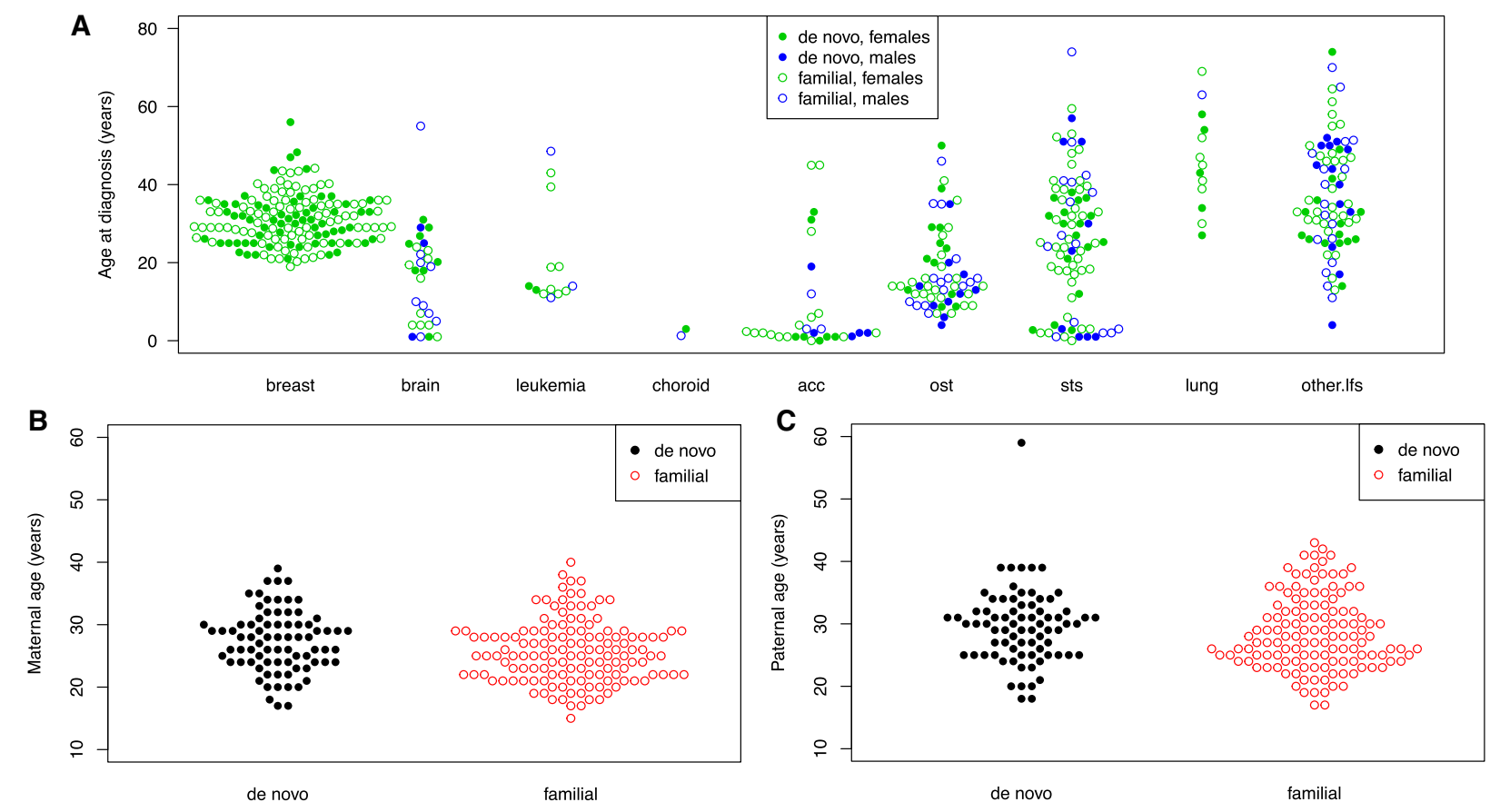

Figure 5. Ages of cancer diagnosis and parental ages in DNM and FM carriers. (A) Ages of cancer diagnosis for all patients. The $x$-axis represents types of different cancer types. Cancers were classified by site: LFS spectrum (osteosarcomas, soft-tissue sarcomas, breast, brain, adrenal, lung cancers, and leukemia) and non-LFS spectrum (prostate, colon, kidney, or thyroid cancers, and others). The green dots are for females, while the blue dots are for males. (B) Distribution of paternal ages. (C) Distribution of maternal ages. All solid dots represent the DNM carriers.

what was reported recently in another study (Renaux-Petel et al. 2018) for deleterious DNMs within TP53.

Supplemental Table S10 summarizes the frequencies of DNMs in each of the four cohorts (MDA, NCI, DFCI, and CHOP) within the validation and discovery sets. In this study of TP53 mutations, there were a total of 49 variants that were recorded in DNMs (Supplemental Table S9) and a total of 82 variants recorded in FMs. Supplemental Figure S2 summarizes the occurrences of DNM and FM variants in the validation and discovery sets, as well as their overlap in mutation types $(24.5 \%$ and $34.1 \%)$. Multiple hotspot mutations were presented in FMs. Among them, two TP53 hotspot mutations (Walerych et al. 2012), NC_000017.11:g.7674220C > T (p.R248Q) and NC_000017.11: g.7673802C $>$ T (p.R273H), were the most enriched in DNMs (p.R248Q, $\mathrm{n}=4$, cancer types: adrenocortical carcinoma, breast, osteosarcoma, soft tissue sarcoma) (Supplemental Table S10). In particular, NC_000017.11:g.7674221G > A (p.R248W) mutations were missing among the DNMs list for this study. Among the FMs, p.R248W was as prevalent as p.R248Q ( $n=7$ vs. 9 out of 242). The observation of $n=0$ out of $82 \mathrm{DNMs}$ for $\mathrm{p} . \mathrm{R} 248 \mathrm{~W}$ was marginally significant $(P$-value $=0.09$, based on a Poisson distribution). The cancer types of FM-p.R248W carriers encompass the cancer spectrum of LFS, including breast cancers. The frequencies of DNMs at different amino acid positions did not differ significantly in the validation (Fig. 6A) and discovery sets (Fig. 6B), supporting the validity of predicted DNMs in the discovery set.

\section{Deleterious DNM status among mutation carriers in BRCAl/2}

We used a data set with 39 extended pedigrees from the Cancer Genetics Network (Anton-Culver et al. 2003): 23 families had $B R C A 1$ mutation carriers and 16 families had BRCA2 mutation car- riers (Supplemental Table S11). Among them, we predicted a total of seven (18\%) DNMs using a cutoff of 0.15 . Among the seven predicted DNMs, three people (13\%) were BRCA1 mutation carriers and four people (25\%) were BRCA2 mutation carriers (Supplemental Fig. S3). Visual inspection of the family cancer history of these seven families (Supplemental Fig. S4) supports the validity of the Famdenovo predictions. The median probability of the 59 predicted FM carriers is 0.005 . Due to a lack of genetic testing information of both parents, we were limited to positive testing results from some parents or siblings, which provided us with only true negatives for validation. A total of 38 people from 15 families were confirmed through parent-child relationship or inferred to be FMs in the case of having multiple siblings testing positive. Famdenovo.BRCA also predicted all of them as FMs (specificity= $100 \%$ ), which demonstrated the accuracy of Famdenovo.BRCA.

\section{Discussion}

Deleterious germline DNMs are more prevalent than previously thought. For cancer genes such as TP53, we estimated a 1:1 ratio of deleterious DNMs compared to FMs in our LFS cohorts, supporting the importance of studying its etiology. To enable future genomic studies, we have developed a statistical method and tool, Famdenovo, to predict the deleterious DNM status in cancer genes using family history data. Famdenovo.TP53 showed excellent performance, an AUC of 0.95 , in discriminating DNM carriers from FM carriers of TP53 deleterious germline mutations in 186 families collected from four clinical cohorts with varying ascertainment criteria. In our case study using 324 germline TP53 pedigrees, Famdenovo.TP53 identified a total of 82 deleterious DNM carriers (increased from only 42 using trio-based genetic testing results, 80 of which were cancer patients) and 450 deleterious FM carriers 
A Histogram of the amino acid change caused by the de novo mutations in the validation set.

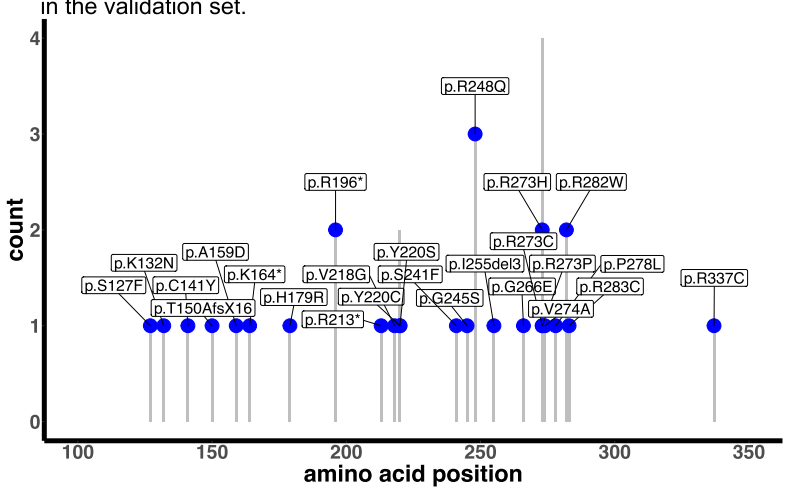

B Histogram of the amino acid change caused by the de novo mutations in the discovery set.

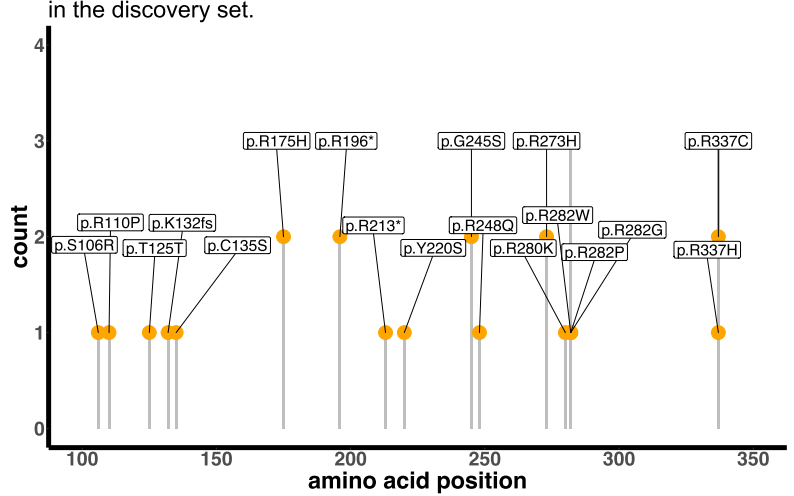

Figure 6. Histograms of DNM frequency at different amino acid positions in the validation and discovery sets. $(A)$ The validation set. $(B)$ The discovery set. The $y$-axis shows the count of DNMs. The $x$-axis shows the amino acid positions.

(317 of which were cancer patients), contributing to a sample size that is large enough to test for associations with patient outcomes: sex, cancer types (more than nine different types), multiple primary cancers, age of cancer diagnosis, parental ages, and mutation types. We provide a reference of cancer spectrum and mutation spectrum to contrast TP53 DNM and FM carriers as a resource for future studies, complementing the ongoing efforts to accurately annotate TP53 variants (Leroy et al. 2017; Tikkanen et al. 2018). Hotspot mutations p.R248Q and p.R273H, the most commonly mutated residues in breast cancer (Walerych et al. 2012), were the most frequently observed mutation types among DNMs. In contrast, p.R248W was not observed in DNMs but was prevalent in FMs.

The observed discrepancy in frequency of p.R248W in DNMs versus FMs, but not in p.R248Q, is consistent with the literature on functional differences in the two mutations (Walerych et al. 2012). When mutated in cancer cells, p.R248W has recently been reported to induce a much stronger response from T cells than p.R248Q (Malekzadeh et al. 2019). Germ cells containing DNMs are sustained in a microenvironment consisting of parental cells that do not carry such DNMs, hence mimicking heterogeneous cellcell interactions that are similar to those presenting somatic mutations. In our data, it is plausible that a neoantigen-like response to p.R248W mutated germ cells has helped destroy these cells so that they are not viable for reproduction. If these germ cells have survived, however, then their offspring may no longer incur such response, as all cells now contain this mutation. This hypothesis is supported by our observation of a high prevalence of p.R248W in FMs. An alternative hypothesis to explain this observation is that DNMs in p.R248W were simply not ascertained by this study, for unknown reasons, as discussed further below.

DNM carriers do not manifest via family history data and are likely to be identified in the clinic only when they have developed multiple cancers, very early-onset breast cancer, or their offspring have developed cancer. They are hence currently underrepresented in cancer genetics studies. We estimate that our four study cohorts may miss up to $45 \%$ of deleterious DNMs in TP53, due to the lack of genetic testing for cancer patients with no family history or healthy individuals who carry these mutations. Correspondingly, we estimate a population prevalence of 0.00076 for deleterious TP53 mutations, which is $26 \%$ higher than shown in previous studies (Peng et al. 2017). Our finding that deleterious DNMs of TP53 are as frequent as FMs in early-onset breast cancer provides strong evidence to support the National Comprehensive Cancer Network (NCCN) guideline for women with early-onset breast cancer to be offered testing for TP53, regardless of family history, to capture the DNM carriers who are not as rare as previously thought. Our results provide a quantitative evaluation on DNMs to genetic counselors so that they may tailor sessions to carefully explain the new knowledge. More importantly, DNMs are sparse in the general population although their downstream effect is detrimental for families. Among the four cohorts, there were 138/324 probands with missing parental genotypes. In these cases, Famdenovo.TP53 would be useful to predict DNMs. Identification of patients with DNMs in order to study them collectively will further our understanding of the molecular mechanism underlying deleterious DNMs, and it is essential for future identification of the hidden DNMs for genetic testing.

Missing data in parental genotypes is more prevalent in HBOC cohorts, which restricts us from conducting an extensive validation of Famdenovo.BRCA. Genetic counselors suggest that it may be due to the higher age of BRCA1/2 mutation carriers at the time they enter a study, resulting in reduced parental followup compared to TP53 mutation carriers (commonly found in childhood cancer patients).

We remain cautious about the downstream interpretations of Famdenovo predicted DNMs and, to a larger extent, about any other biological factors that may confound our interpretation. We enumerate the following five considerations in the context of TP53, which are applicable to BRCA1/2 and other future genes of interest. First, mosaicism in parents could be partially contributing to DNMs in the children (Renaux-Petel et al. 2018). If a parent presents an LFS-like phenotype due to a mosaic TP53 mutation, Famdenovo.TP53 would consider the parent as a germline mutation carrier, hence deducing the child to be an FM carrier. The DNM status of a child under this condition would then be missed by Famdenovo.TP53. Such a condition is rare, at $~ 5 \%$ (RenauxPetel et al. 2018), and of less concern to clinical practice, as this population is already targeted by genetic testing. Patients with an LFS-like phenotype are often tested, first with Sanger sequencing, then with NGS at high depth, until mosaicism is confirmed. Second, somatic clonal expansion in cancer patients may create a look-alike germline TP53 mutation, resulting in a false positive in DNM status. However, such observation was reported in a general panel-testing population, which is supposedly $>99.9 \%$ without TP53 mutations, hence presenting an expected low positive predictive value (Weitzel et al. 2018). By focusing on clinically ascertained families, we do not expect aberrant clonal expansion to be the cause of the identified DNMs. Furthermore, we only observed

\section{Genome Research}

www.genome.org 
two DNM carriers who have ever had leukemia, a cancer type known to present clonal hematopoiesis. Third, Famdenovo.TP53 assumes the mutations are deleterious and their effect on cancer outcomes follow the penetrance curves previously estimated from a set of families. Variants with unknown significance in TP53 were excluded from the study. Fourth, DNMs across the genome were reported to be shared in siblings (Jónsson et al. 2018). Within a single gene like TP53, however, we did not observe sibling sharing. Last, cutoffs for DNM probabilities to classify the mutation carriers into DNM and FM carriers were determined on the same data set. Future validation studies are needed to determine one or several recommended cutoffs for clinical decisionmaking.

Because of our study design, distinct from existing DNM studies that are genome-wide and across many individuals, we are limited to a small number of mutations, which, even with the addition of the Famdenovo's discovery set, would not be sufficient to perform sequence context-based analyses that would follow those existing DNM studies. In contrast, what existing studies cannot provide is Famdenovo's laser-beam view of the impact of de novo mutations in a single gene and a single disease syndrome. Both study designs should be carried out in order to provide a well-rounded understanding of de novo mutations.

In summary, we present a new epidemiological study design, enabled by a statistical method called Famdenovo, to evaluate the clinical impact of deleterious de novo mutations in cancer genes. We introduce both Famdenovo.TP53 and Famdenovo.BRCA and illustrate both models using family history cohorts. This study design is important for future genetic research, as well as the clinical management of patients and their families as we learn new biology and associated clinical risk for DNM carriers. Famdenovo was developed as a freely available R package and Shiny web app (http ://bioinformatics.mdanderson.org/public-software/famdenovo).

\section{Methods}

\section{Model development}

Famdenovo estimates the probability of de novo mutation in any designated family member who carries a deleterious germline mutation in a given gene, based on a detailed family disease history and values of genetic parameters, such as the penetrance and prevalence, for the gene and the corresponding diseases. The prevalence for gene mutations is expressed as $\operatorname{Pr}(G)$, where $G$ denotes genotype, which could be wild type (denoted as 0 ), a heterozygous mutation (denoted as 1), or a homozygous mutation (denoted as 2). $\operatorname{Pr}(G)$ for three genotypes can be derived from the prevalence of the mutated alleles using the Hardy-Weinberg equilibrium. The penetrance is the probability of developing the disease at a given age for individuals who carry the germline mutations. The disease of interest is an inherited syndrome, such as Li-Fraumeni syndrome or HBOC. The genes (one or more) of interest are those that are known to be the major cause of the corresponding inherited syndrome when they are mutated. The germline mutations here correspond to deleterious mutations that are accepted in clinics as mutations that would categorize the carriers as high risk for developing the disease in the future and who will be suggested to adhere to a series of cancer prevention protocols. Other mutations in these genes, including those that are variants of unknown significance, are considered as wild type in the model. Either the dominant or recessive mode of inheritance can be assumed in Famdenovo.

Let $P$ be the pedigree information of the family (e.g., the pedigree drawn in Fig. 1), let $\boldsymbol{D}$ be the disease phenotype information of all family members, and $G_{C}$ be the genotype of the carrier, then the probability of the mutation being de novo can be written as $\operatorname{Pr}\left(G_{c}\right.$ is de novo $\mid G_{c}$ is germline, $\left.\mathbf{D}, \mathbf{P}\right)$, referred to as the "de novo probability."

To calculate $\operatorname{Pr}\left(G_{c}\right.$ is de novo $\mid G_{c}$ is germline, $\left.\mathbf{D}, \mathbf{P}\right)$, we have the following equation:

$$
\begin{gathered}
\operatorname{Pr}\left(G_{c} \text { is de novo } \mid G_{c} \text { is germline, } \mathbf{D}, \mathbf{P}\right) \\
=\operatorname{Pr}\left(G_{m}=0, G_{f}=0 \mid G_{c}=1, \mathbf{D}, \mathbf{P}\right) \\
=\frac{\operatorname{Pr}\left(G_{c}=1 \mid G_{m}=0, G_{f}=0, \mathbf{D}, \mathbf{P}\right) \times \operatorname{Pr}\left(G_{f}=0 \mid \mathbf{D}, \mathbf{P}\right) \times \operatorname{Pr}\left(G_{m}=0 \mid \mathbf{D}, \mathbf{P}\right)}{\operatorname{Pr}\left(G_{c}=1 \mid \mathbf{D}, \mathbf{P}\right)}
\end{gathered}
$$

where $G_{m}$ is the genotype of the mother and $G_{f}$ is the genotype of the father. In Equation 1, all four probabilities are usually difficult to obtain through direct calculations. Hence, we apply Mendelian models to derive them. Let the family cancer history $\mathbf{H}=(\mathbf{P}, \mathbf{D})$. In a Mendelian model, the probability of the person's genotype given the family cancer history $\operatorname{Pr}\left(G_{0} \mid \mathbf{H}\right)$ is the updated population prevalence $\operatorname{Pr}\left(G_{0}\right)$ by incorporating family cancer history $\boldsymbol{H}$. Here, $G_{0}$ denotes the genotype of the person of interest, which can be $G_{m}$, $G_{f}$, or $G_{c}$. We can estimate it via the following formula:

$$
\begin{gathered}
\operatorname{Pr}\left(G_{0} \mid \mathbf{H}\right)=\operatorname{Pr}\left(G_{0} \mid \mathrm{H}_{0}, \quad \mathrm{H}_{1}, \ldots, \mathrm{H}_{\mathrm{n}}\right) \\
=\frac{\operatorname{Pr}\left(G_{0}\right) \operatorname{Pr}\left(\mathbf{H} \mid G_{0}\right)}{\sum G_{0} \operatorname{Pr}\left(G_{0}\right) \operatorname{Pr}\left(\mathbf{H} \mid G_{0}\right)} \\
\operatorname{Pr}\left(\mathbf{H} \mid G_{0}\right)=\sum_{G_{1}, G_{2}, \ldots, G_{n}} \operatorname{Pr}(\mathbf{H} \mid \boldsymbol{G}) \operatorname{Pr}\left(G_{1}, G_{2}, \ldots, G_{n} \mid G_{0}\right)= \\
\sum_{G_{1}, G_{2}, \ldots, G_{n}}\left[\prod_{i=0}^{n} \operatorname{Pr}\left(\mathrm{H}_{i} \mid G_{i}\right)\right] \operatorname{Pr}\left(G_{1}, G_{2}, \ldots, G_{n} \mid G_{0}\right) .
\end{gathered}
$$

In Equations 2 and 3, $n$ is the total number of the counselee's relatives within a family. $\operatorname{Pr}\left(\mathbf{H} \mid G_{0}\right)$ is the probability of the phenotypes for the whole pedigree given the genotype of the counselee, which is the weighted average of the probabilities of family history given each possible genotype configuration of all relatives $\operatorname{Pr}(\mathbf{H} \mid \mathbf{G})$. The weights are the probabilities of the genotype configuration based on Mendelian transmission. $\operatorname{Pr}(\mathbf{H} \mid \mathbf{G})$ are products of the individual probability distributions of penetrance $\operatorname{Pr}\left(H_{i} \mid G_{i}\right)$ when we assume conditional independence. With the equations above for $\operatorname{Pr}\left(G_{0} \mid \mathbf{H}\right)$, we can then calculate the four probabilities in Equation 1, respectively, by assigning the right person as $G_{0}$ and assuming a known genotype status in the parents, when needed.

The posterior probability calculation is performed using the Elston-Stewart peeling algorithm. This algorithm characterizes Mendelian transmission using a transmission matrix of the probability of the genotype for an individual, given the genotypes of the father and mother, $\operatorname{Pr}\left(G_{i} \mid G_{f i}, G_{m i}\right)$ (Fernando et al. 1993).

With the calculated probabilities, we classify the DNM status based on a cutoff on the probability. $\operatorname{Pr}\left(G_{c}\right.$ is de novo $\mid G_{c}$ is germline, $\left.\mathbf{D}, \mathbf{P}\right) \geq$ cutoff are identified as DNM carriers. We used the validation data in this study to determine an ideal cutoff.

For Famdenovo.TP53, we used a previous penetrance estimate for TP53 mutation carriers and noncarriers from six large pediatric sarcoma families at MD Anderson, which are not included in this study (Wu et al. 2006). We assumed the TP53 mutation follows the Hardy-Weinberg equilibrium, but this could be modified by user input when homozygous genotype information is published. The mutation prevalence, i.e., allele frequency, is specified as 0.0006 for pathogenic TP53 mutations, which was derived in our previous study (Peng et al. 2017). The assumed frequencies for the three genotypes (homozygous reference, heterozygous, and homozygous variant) were 0.9988, 0.001199, and $3.6 \times 10^{-07}$, 
respectively. We used $20 \%$ as percent DNMs among all mutations. The allele frequency and DNM rate are priors that are then updated by family history to generate a posterior probability. Both penetrance and prevalence estimates were previously validated using external study cohorts that are different from those in this study (Peng et al. 2017).

For Famdenovo.BRCA, we used previous externally validated penetrance estimates of $B R C A 1$ and $B R C A 2$ mutations of the U.S. population for both Ashkenazi Jewish (AJ) and non-AJ families (Chen et al. 2006a; Parmigiani et al. 2007). We used 0.00305 and 0.0034 as the mutation allele frequencies for BRCA1 and $B R C A 2$, respectively. We assumed a $15 \%$ DNM rate among all mutations. The allele frequency and DNM rate are priors that were then updated by family history to generate a posterior probability. The same cutoff value of 0.15 was used to classify DNMs in $B R C A 1 / 2$.

\section{Study cohorts}

We evaluated our method on TP53 using four cohorts (Supplemental Table S12). (A) Families with LFS primarily ascertained through clinical criteria were included in the MD Anderson cohort. Details on data collection, specific cancers, and germline testing for this cohort have been published (Shin et al. 2020). This cohort has 82 families with known de novo status and 58 families with unknown de novo status. (B) The National Cancer Institute (NCI) LFS cohort (NCT01443468), our second cohort, is from a long-term prospective, natural history study that started in 2011 (Mai et al. 2016). The cohort includes individuals meeting classic or Li-Fraumenilike diagnostic criteria, having a pathogenic germline TP53 mutation or a first- or second-degree relative with a TP53 mutation, or having a personal history of choroid plexus carcinoma, adrenocortical carcinoma, or at least three primary cancers (Birch et al. 1994). This cohort includes 66 families with known de novo status and 12 families with unknown de novo status. (C) The DFCI LFS cohort, our third cohort, is comprised of patients identified in the course of clinical genetics practice. The early years of the cohort were the result of single gene testing of individuals with features suggestive of LFS. Since 2012, the cohort has included individuals with TP53 mutations identified on multigene panel testing and individuals referred for clinical consultation of enrollment in a wholebody MRI surveillance protocol. This cohort has 30 families with known de novo status and 61 families with unknown de novo status. (D) Data from the CHOP were obtained through the Cancer Predisposition Program, which provides care and counseling for children with a genetic predisposition to cancer. This cohort has eight families with known de novo status and seven families with unknown de novo status.

We also tested Famdenovo.BRCA using a small set of family data that is from the Cancer Genetics Network (http://www .cancergen.org/studies.shtml) (Anton-Culver et al. 2003), a network established by NCI in 1999 to explore the impact of genetics on cancer susceptibility. Individuals with a family history of cancer were enrolled at clinical centers across the United States and completed questionnaires regarding family history of cancer and other relevant information. Most families were ascertained through high-risk clinics and have a family history of breast or ovarian cancers.

\section{Mutation testing}

For the MD Anderson cohort, peripheral blood samples were collected after informed consents were obtained. The probands' TP53 mutation status was determined by PCR sequencing of exons 2-11 (Hwang et al. 2003). At the MD Anderson Cancer
Center, when a TP53 mutation was identified, all first-degree relatives of the proband (affected and unaffected by cancer) and any other family member at risk of carrying the familial mutation were tested. Extending germline testing based on mutation status and not on phenotype of family members should not introduce ascertainment bias during analysis (Katki et al. 2008). Individuals unavailable for testing (largely deceased) linked to mutation carriers were considered as obligate mutation carriers. No other family member was tested when the proband tested negative.

For the NCI cohort, copies of the clinical TP53 test reports were obtained and verified by the study team for those tested prior to enrollment. For individuals actively participating in the protocol and not previously tested, clinical genetic testing was performed after enrollment. All at-risk family members of individuals who tested positive for a mutation (either prior to enrollment or on study) were offered the option of having site-specific testing through the study. No testing was offered to relatives if the proband tested negative for a TP53 mutation. Additionally, high resolution melt analysis and multiplex ligation-dependent probe amplification were performed in the NCI cohort to detect large deletions or genomic rearrangements.

For the DFCI cohort, the patient information was collected by searching through the Clinical Operations and Research Information System (CORIS) database at the DFCI. The classic (Li and Fraumeni 1969) and updated Chompret (Tinat et al. 2009) criteria were applied for selecting eligible families. The methods for TP53 testing were further described in a previous study from DFCI (Rath et al. 2013).

For the CHOP cohort, pediatric oncology patients were evaluated in the Cancer Predisposition Program based on a primary tumor type concordant with the LFS tumor spectrum (e.g., adrenocortical carcinoma, choroid plexus carcinoma, early onset rhabdomyosarcoma, multiple primary cancers, etc.) and/or a suggestive family cancer history. Most patients met classic or Chompret criteria. The laboratories used to complete clinical TP53 testing included the Genetic Diagnostic Laboratory at the University of Pennsylvania, The Hospital for Sick Children, or Ambry Genetics.

\section{Model evaluation and comparison}

In four cohorts (described above) of TP53 mutation carriers with known de novo status, we used Famdenovo.TP53 to calculate the de novo probability for each individual. Because mutation carriers within the same family are not independent, we used family-wise de novo probability and status for model evaluation. If a family had at least one family member with a de novo probability that was over the cutoff, they were defined as a de novo family; otherwise they were defined as a familial family.

Validation was performed on 186 families with known DNM status from the four cohorts. We used OEs to evaluate the calibration and ROC curves to evaluate our model's discrimination ability. The $\mathrm{OE}$ is the ratio between the observed number of de novo TP53 mutations and the summation of the estimated probabilities of de novo TP53 mutations; ideally, the observed number of DNMs equals the estimated number $(\mathrm{OE}=1)$. The ROC curve is generated by plotting the true positive rate against the false positive rate at various prediction cutoffs using Famdenovo.TP53. A high area under the ROC curve (AUC), i.e., concordance index, indicates that we can find a point on the ROC curve for determining the de novo status with a high true positive rate and a low false positive rate.

We also compared our method with the other two criterionbased prediction models. The partial AUC is rescaled to the full

\section{Genome Research}

www.genome.org 
range of 0 to 1 using the following equation:

Rescaled AUC of model $=\frac{\text { partial AUC of model }}{\text { partial AUC of Famdenovo }} *$ full AUC of Famdenovo

Finally, 95\% CIs were obtained by bootstrapping the AUC values of Famdenovo and the AUC or rescaled AUC of the two criterion-based models. All data analyses were performed in R.3.6.2 (R Core Team 2019).

\section{Data access}

The TP53 mutation and cancer data from all studies are provided in Supplemental Table S1. The BRCA1/2 mutation and cancer data from CGN are provided in Supplemental Table S11. The R package Famdenovo_0.1.1.tar.gz and scripts for Famdenovo.BRCA are provided in the Supplemental Materials. Integration of Famdenovo.BRCA into the Famdenovo $\mathrm{R}$ package will be made in the future after further testing in larger study cohorts.

The complete family history data for TP53 from all studies have been submitted to the LiFE consortium. To request access, use the following contact information: MD Anderson Cancer Center: Dr. Louise Strong at lstrong@mdanderson.org and Dr. Wenyi Wang at wwang7@mdanderson.org; the National Cancer Institute: Dr. Sharon Savage at savagesh@mail.nih.gov; Dana Farber Cancer Institute: Dr. Judy Garber at Judy_Garber@dfci.harvard.edu; and Children's Hospital of Philadelphia: Kristin Zelley at zelleyk@email.chop.edu. The complete family history data for BRCA1/2 have been submitted to the Cancer Genetics Network. To request access, contact Dr. Dianne Finkelstein at dfinkelstein@mgh.harvard.edu.

\section{Competing interest statement}

D.B. coleads the BayesMendel laboratory, which licenses software for the computation of risk prediction models.

\section{Acknowledgments}

We thank Dr. Chris Amos and Dr. Banu Arun for their constructive comments on this manuscript. We thank Timothy Cheng and Jessica Swann for reading the manuscript and providing word editing. We thank the reviewers for their constructive comments which have substantially improved the paper. The work of X.P. and W.W. are supported in part by National Institutes of Health (NIH) R01CA158113. E.B.D-E., F G., and W.W. are supported in part by NIH R01CA183793 and R01CA239342. The work of W.W. is also supported in part by NIH P30CA016672. The work of S.A.S. is supported by the intramural research program of the Division of Cancer Epidemiology and Genetics, National Cancer Institute. J.B. and L.C.S. are supported in part by the NIH P01CA34936. The work of D.B. is supported in part by the NIH R01CA207365, R01CA239342. The CGN data collection and curation are supported by N01PC74400-6-0-1.

Author contributions: Concept and design: F.G., X.P., and W.W.; development of methodology: F.G., X.P., and W.W.; acquisition of data: J.B., L.C.S., P.L.M., S.A.S., K.Z., K.E.N., and J.G.; analysis and interpretation of data: F.G., X.P., E.B.D.-E., C.V.R., M.D.M, D.B., V.E.J., and W.W.; writing, review, and/or revision of the manuscript: F.G., X.P., E.B.D.-E., C.V.R., J.B., D.B., P.L.M., V.E.J., K.Z., K.E.N., J.E.G., S.A.S., L.C.S., and W.W.; administrative, technical, or material support: E.B.D.-E.; study supervision: W.W.

\section{References}

Acuna-Hidalgo R, Veltman JA, Hoischen A. 2016. New insights into the generation and role of de novo mutations in health and disease. Genome Biol 17: 241. doi:10.1186/s13059-016-1110-1

Anton-Culver H, Ziogas A, Bowen D, Finkelstein D, Griffin C, Hanson J, Isaacs C, Kasten-Sportes C, Mineau G, Nadkarni P, et al. 2003. The Cancer Genetics Network: recruitment results and pilot studies. Community Genet 6: 171-177. doi:10.1159/000078165

Battle A, Montgomery SB. 2014. Determining causality and consequence of expression quantitative trait loci. Hum Genet 133: 727-735. doi:10 .1007/s00439-014-1446-0

Birch JM, Hartley AL, Tricker KJ, Prosser J, Condie A, Kelsey AM, Harris M, Jones PH, Binchy A, Crowther D, et al. 1994. Prevalence and diversity of constitutional mutations in the p53 gene among $21 \mathrm{Li}$-Fraumeni families. Cancer Res 54: 1298-1304.

Chen S, Wang W, Broman KW, Katki HA, Parmigiani G. 2004. BayesMendel: an R environment for Mendelian risk prediction. Stat Appl Genet Mol Biol 3: 21. doi:10.2202/1544-6115.1063

Chen S, Iversen ES, Friebel T, Finkelstein D, Weber BL, Eisen A, Peterson LE, Schildkraut JM, Isaacs C, Peshkin BN, et al. 2006a. Characterization of BRCA1 and BRCA2 mutations in a large United States sample. J Clin Oncol 24: 863-871. doi:10.1200/JCO.2005.03.6772

Chen S, Wang W, Lee S, Nafa K, Lee J, Romans K, Watson P, Gruber SB, Euhus D, Kinzler KW, et al. 2006b. Prediction of germline mutations and cancer risk in the Lynch syndrome. JAMA 296: 1479-1487. doi:10.1001/jama.296.12.1479

Conrad DF, Jakobsson M, Coop G, Wen X, Wall JD, Rosenberg NA, Pritchard JK. 2006. A worldwide survey of haplotype variation and linkage disequilibrium in the human genome. Nat Genet 38: 1251-1260. doi:10 $1038 /$ ng1911

Correa H. 2016. Li-Fraumeni syndrome. J Pediatr Genet 05: 084-088. doi:10 $.1055 / \mathrm{s}-0036-1579759$

de Ligt J, Willemsen MH, van Bon BWM, Kleefstra T, Yntema HG, Kroes T, Vulto-van Silfhout AT, Koolen DA, de Vries P, Gilissen C, et al. 2012. Diagnostic exome sequencing in persons with severe intellectual disability. N Engl J Med 367: 1921-1929. doi:10.1056/NEJMoa1206524

De Rubeis S, He X, Goldberg AP, Poultney CS, Samocha K, Cicek AE, Kou Y, Liu L, Fromer M, Walker S, et al. 2014. Synaptic, transcriptional and chromatin genes disrupted in autism. Nature 515: 209-215. doi:10 .1038 /nature 13772

Dryja TP, Morrow JF, Rapaport JM. 1997. Quantification of the paternal allele bias for new germline mutations in the retinoblastoma gene. Hum Genet 100: 446-449. doi:10.1007/s004390050531

Euhus DM, Smith KC, Robinson L, Stucky A, Olopade OI, Cummings S, Garber JE, Chittenden A, Mills GB, Rieger P, et al. 2002. Pretest prediction of BRCA1 or BRCA2 mutation by risk counselors and the computer model BRCAPRO. J Natl Cancer Inst 94: 844-851. doi:10.1093/jnci/94 .11 .844

Evans DG, Howard E, Giblin C, Clancy T, Spencer H, Huson SM, Lalloo F. 2010. Birth incidence and prevalence of tumor-prone syndromes: estimates from a UK family genetic register service. Am J Med Genet A 152A: 327-332. doi:10.1002/ajmg.a.33139

Fernando RL, Stricker C, Elston RC. 1993. An efficient algorithm to compute the posterior genotypic distribution for every member of a pedigree without loops. Theor Appl Genet Int J Plant Breed Res 87: 89-93. doi:10 .1007/BF00223750

Francioli LC, Polak PP, Koren A, Menelaou A, Chun S, Renkens I, van Duijn CM, Swertz M, Wijmenga C, van Ommen G, et al. 2015. Genome-wide patterns and properties of de novo mutations in humans. Nat Genet 47: 822-826. doi:10.1038/ng.3292

Goldmann JM, Veltman JA, Gilissen C. 2019. De novo mutations reflect development and aging of the human germline. Trends Genet 35: 828-839. doi:10.1016/j.tig.2019.08.005

Gonzalez KD, Buzin CH, Noltner KA, Gu D, Li W, Malkin D, Sommer SS. 2009. High frequency of de novo mutations in Li-Fraumeni syndrome. J Med Genet 46: 689-693. doi:10.1136/jmg.2008.058958

Hwang SJ, Lozano G, Amos CI, Strong LC. 2003. Germline p53 mutations in a cohort with childhood sarcoma: sex differences in cancer risk. Am J Hum Genet 72: 975-983. doi:10.1086/374567

Iossifov I, O'Roak BJ, Sanders SJ, Ronemus M, Krumm N, Levy D, Stessman HA, Witherspoon KT, Vives L, Patterson KE, et al. 2014. The contribution of de novo coding mutations to autism spectrum disorder. Nature 515: 216-221. doi: $10.1038 /$ nature 13908

Jett K, Friedman JM. 2010. Clinical and genetic aspects of neurofibromatosis 1. Genet Med 12: 1-11. doi:10.1097/GIM.0b013e3181bf15e3

Jónsson H, Sulem P, Arnadottir GA, Pálsson G, Eggertsson HP, Kristmundsdottir S, Zink F, Kehr B, Hjorleifsson KE, Jensson B, et al. 2018. Multiple transmissions of de novo mutations in families. Nat Genet 50: 1674-1680. doi:10.1038/s41588-018-0259-9 
Katki HA, Blackford A, Chen S, Parmigiani G. 2008. Multiple diseases in carrier probability estimation: accounting for surviving all cancers othe than breast and ovary in BRCAPRO. Stat Med 27: 4532-4548. doi:10 $.1002 / \operatorname{sim} .3302$

Kondrashov AS. 2003. Direct estimates of human per nucleotide mutation rates at 20 loci causing Mendelian diseases. Hum Mutat 21: 12-27. doi:10.1002/humu.10147

Kong A, Frigge ML, Masson G, Besenbacher S, Sulem P, Magnusson G, Gudjonsson SA, Sigurdsson A, Jonasdottir A, Wong WS, et al. 2012 Rate of de novo mutations and the importance of father's age to disease risk. Nature 488: 471-475. doi:10.1038/nature11396

Krumm N, Turner TN, Baker C, Vives L, Mohajeri K, Witherspoon K, Raja A, Coe BP, Stessman HA, He ZX, et al. 2015. Excess of rare, inherited truncating mutations in autism. Nat Genet 47: 582-588. doi:10.1038/ng .3303

Leroy B, Ballinger ML, Baran-Marszak F, Bond GL, Braithwaite A, Concin N, Donehower LA, El-Deiry WS, Fenaux P, Gaidano G, et al. 2017. Recommended guidelines for validation, quality control, and reporting of TP53 variants in clinical practice. Cancer Res 77: 1250-1260. doi:10 .1158/0008-5472.CAN-16-2179

Li FP, Fraumeni JF Jr. 1969. Soft-tissue sarcomas, breast cancer, and other neoplasms. A familial syndrome? Ann Intern Med 71: 747-752. doi:10 .7326/0003-4819-71-4-747

Lipson M, Loh PR, Sankararaman S, Patterson N, Berger B, Reich D. 2015 Calibrating the human mutation rate via ancestral recombination density in diploid genomes. PLoS Genet 11: e1005550. doi:10.1371/journal .pgen. 1005550

Lynch M. 2010. Rate, molecular spectrum, and consequences of human mutation. Proc Natl Acad Sci 107: 961-968. doi:10.1073/pnas .0912629107

Mai PL, Best AF, Peters JA, DeCastro RM, Khincha PP, Loud JT, Bremer RC, Rosenberg PS, Savage SA. 2016. Risks of first and subsequent cancers among TP53 mutation carriers in the National Cancer Institute LiFraumeni syndrome cohort. Cancer 122: 3673-3681. doi: $10.1002 / \mathrm{cncr}$ .30248

Malekzadeh P, Rosenberg SA, Drew C, Invest JC, Malekzadeh P, Pasetto A Robbins PF, Parkhurst MR, Paria BC, Jia L, et al. 2019. Neoantigen screening identifies broad TP53 mutant immunogenicity in patients with epithelial cancers. J Clin Invest 129: 1109-1114. doi:10.1172/ JCI123791

Malkin D, Li FP, Strong LC, Fraumeni JF Jr, Nelson CE, Kim DH, Kassel J, Gryka MA, Bischoff FZ, Tainsky MA, et al. 1990. Germ line p53 mutations in a familial syndrome of breast cancer, sarcomas, and other neoplasms. Science 250: 1233-1238. doi:10.1126/science.1978757

Michaelson JJ, Shi Y, Gujral M, Zheng H, Malhotra D, Jin X, Jian M, Liu G, Greer D, Bhandari A, et al. 2012. Whole-genome sequencing in autism identifies hot spots for de novo germline mutation. Cell 151: 14311442. doi:10.1016/j.cell.2012.11.019

Nachman MW, Crowell SL. 2000. Estimate of the mutation rate per nucleotide in humans. Genetics 156: 297-304.

Nichols KE, Malkin D, Garber JE, Fraumeni J, Li FP. 2001. Germ-line p53 mutations predispose to a wide spectrum of early-onset cancers. Cancer Epidemiol Biomarkers Prev 10: 83-87.

Olivier M, Goldgar DE, Sodha N, Ohgaki H, Kleihues P, Hainaut P, Eeles RA. 2003. Li-Fraumeni and related syndromes. Cancer Res 63: 6643-6650.

Parmigiani G, Berry D, Aguilar O. 1998. Determining carrier probabilities for breast cancer-susceptibility genes BRCA1 and BRCA2. Am J Hum Genet 62: 145-158. doi:10.1086/301670

Parmigiani G, Chen S, Iversen ES Jr, Friebel TM, Finkelstein DM, AntonCulver H, Ziogas A, Weber BL, Eisen A, Malone KE, et al. 2007. Validity of models for predicting BRCA1 and BRCA2 mutations. Ann Intern Med 147: 441-450. doi:10.7326/0003-4819-147-7-20071002000002
Peng G, Bojadzieva J, Ballinger ML, Li J, Blackford AL, Mai PL, Savage SA Thomas DM, Strong LC, Wang W. 2017. Estimating TP53 mutation carrier probability in families with Li-Fraumeni syndrome using LFSPRO. Cancer Epidemiol Biomarkers Prev 26: 837-844. doi:10.1158/1055-9965 .EPI-16-0695

Rahbari R, Wuster A, Lindsay SJ, Hardwick RJ, Alexandrov LB, Al Turki S, Dominiczak A, Morris A, Porteous D, Smith B, et al. 2016. Timing, rates and spectra of human germline mutation. Nat Genet 48: 126-133. doi:10.1038/ng.3469

Rath MG, Masciari S, Gelman R, Miron A, Miron P, Foley K, Richardson AL, Krop IE, Verselis SJ, Dillon DA, et al. 2013. Prevalence of germline TP53 mutations in HER2+ breast cancer patients. Breast Cancer Res Treat 139: 193-198. doi:10.1007/s10549-012-2375-z

R Core Team. 2019. $R$ : a language and environment for statistical computing. $\mathrm{R}$ Foundation for Statistical Computing, Vienna. https://www.R-pro ject.org/.

Renaux-Petel M, Charbonnier F, Théry JC, Fermey P, Lienard G, Bou J, Coutant S, Vezain M, Kasper E, Fourneaux S, et al. 2018. Contribution of de novo and mosaic TP53 mutations to Li-Fraumeni syndrome. $J$ Med Genet 55: 173-180. doi:10.1136/jmedgenet-2017-104976

Robinson EB, Samocha KE, Kosmicki JA, McGrath L, Neale BM, Perlis RH, Daly MJ. 2014. Autism spectrum disorder severity reflects the average contribution of de novo and familial influences. Proc Natl Acad Sci 111: 15161-15165. doi:10.1073/pnas.1409204111

Shin SJ, Dodd-Eaton EB, Gao F, Bojadzieva J, Chen J, Kong X, Amos CI, Ning J, Strong LC, Wang W. 2020. Penetrance estimates over time to first and second primary cancer diagnosis in families with Li-Fraumeni syndrome: a single institution perspective. Cancer Res 80: 347-353. doi:10 .1158/0008-5472.CAN-19-0725

Tikkanen T, Leroy B, Fournier JL, Risques RA, Malcikova J, Soussi T. 2018. Seshat: a web service for accurate annotation, validation, and analysis of TP53 variants generated by conventional and next-generation sequencing. Hum Mutat 39: 925-933. doi:10.1002/humu.23543

Tinat J, Bougeard G, Baert-Desurmont S, Vasseur S, Martin C, Bouvignies E, Caron O, Paillerets BB, Berthet P, Dugast C, et al. 2009. 2009 Version of the chompret criteria for Li Fraumeni syndrome. J Clin Oncol 27: e108e109. doi:10.1200/JCO.2009.22.7967

Walerych D, Napoli M, Collavin L, Del Sal G. 2012. The rebel angel: mutant p53 as the driving oncogene in breast cancer. Carcinogenesis 33: 20072017. doi:10.1093/carcin/bgs232

Wang W, Chen S, Brune KA, Hruban RH, Parmigiani G, Klein AP. 2007. PancPRO: risk assessment for individuals with a family history of pancreatic cancer. J Clin Oncol 25: 1417-1422. doi:10.1200/JCO.2006.09 .2452

Wang W, Niendorf KB, Patel D, Blackford A, Marroni F, Sober AJ, Parmigiani G, Tsao H. 2010. Estimating CDKN2A carrier probability and personalizing cancer risk assessments in hereditary melanoma using MelaPRO. Cancer Res 70: 552-559. doi:10.1158/0008-5472.CAN-09-2653

Weitzel JN, Chao EC, Nehoray B, Van Tongeren LR, LaDuca H, Blazer KR, Slavin T, DABMD FACMG, Pesaran T, Rybak C, et al. 2018. Somatic TP53 variants frequently confound germ-line testing results. Genet Med 20: 809-816. doi:10.1038/gim.2017.196

Wong WSW, Solomon BD, Bodian DL, Kothiyal P, Eley G, Huddleston KC, Baker R, Thach DC, Iyer RK, Vockley JG, et al. 2016. New observations on maternal age effect on germline de novo mutations. Nat Commun 7: 10486. doi:10.1038/ncomms10486

Wu CC, Shete S, Amos CI, Strong LC. 2006. Joint effects of germ-line p53 mutation and sex on cancer risk in Li-Fraumeni syndrome. Cancer Res 66: 8287-8292. doi:10.1158/0008-5472.CAN-05-4247

Received May 4, 2019; accepted in revised form June 25, 2020.

\section{Genome Research}

www.genome.org 


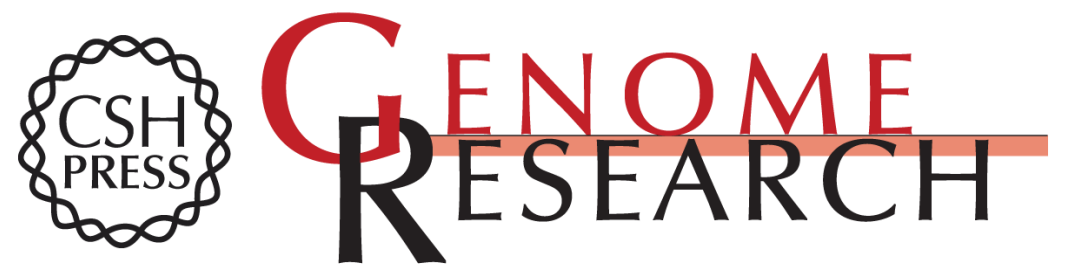

\section{A pedigree-based prediction model identifies carriers of deleterious de novo mutations in families with Li-Fraumeni syndrome}

Fan Gao, Xuedong Pan, Elissa B. Dodd-Eaton, et al.

Genome Res. 2020 30: 1170-1180 originally published online August 18, 2020

Access the most recent version at doi:10.1101/gr.249599.119

Supplemental Material

References

Open Access

Creative Commons

License

Email Alerting Service
http://genome.cshlp.org/content/suppl/2020/08/18/gr.249599.119.DC1

This article cites 53 articles, 17 of which can be accessed free at: http://genome.cshlp.org/content/30/8/1170.full.html\#ref-list-1

Freely available online through the Genome Research Open Access option.

This article, published in Genome Research, is available under a Creative Commons License (Attribution-NonCommercial 4.0 International), as described at http://creativecommons.org/licenses/by-nc/4.0/.

Receive free email alerts when new articles cite this article - sign up in the box at the top right corner of the article or click here.

\section{Affordable, Accurate Sequencing.}

